\title{
An Innovative EPW Design Using Add-on Features to Meet Malaysian Requirements
}

\author{
Mohammed Hayyan Alsibai \\ Faculty of Engineering Technology, \\ University Malaysia Pahang, \\ Gambang, Kuantan, Malaysia \\ mhdhayyan@gmail.com
}

\author{
Mhd Saeed Sharif \\ School of Architecture, Computing and \\ Engineering, \\ UEL, E16 2RD, London, UK. \\ s.sharif@uel.ac.uk
}

\author{
Salma Yaakub and Nurul Nadia \\ Nor Hamran \\ Ftek, University Malaysia Pahang, \\ Gambang, Kuantan, Malaysia \\ salmayaakub94@gmail.com, \\ nnadiahamran@ump.edu.my
}

\begin{abstract}
Electric Powered Wheelchair (EPW) is a special Electric Vehicle (EV). It is used by senior citizens, handicapped, disabled, people with mobility impairment or people who have health complaints. Therefore, it is not always safe to use an EPW independently as users are more subject to fatigue, weakness and emergency situations. Due to the special needs of EPW drivers/users, the design of the EPW and its controlling system should fulfill their requirements. This paper proposes a new design for EPW which is suitable for Malaysian community needs. The design takes in consideration the easiness of the independent use, the price reduction and the flexibility in changing the controlling method. A smartphone is used as an add-on controlling option besides to the normal joystick. A health monitoring system which implements Internet of Things (IoT) features is also presented as an add-on device. The EPW system is designed to be extendable and accepts other add-on devices. The system is tested in real modes and it is validated as a realtime system.
\end{abstract}

Index Terms - EV, Assistive technology, IoT, embedded system, wheelchair.

\section{INTRODUCTION}

The term "disability" represents different meanings to different individuals. It can be defined as total or partial loss of a part of the body or a mental function. It also may mean the malfunction of the body part or the function [1]. In Malaysian "Persons with Disabilities act 2008", disabled people are defined as those who "have long term physical, mental, intellectual or sensory impairments which in interaction with various barriers may hinder their full and effective participation in society".

According to Malaysian national news agency in 2009, 1.3 million people, i.e. $4.6 \%$ of the Malaysian population, have disabilities [1]. United Nations Children's Fund (UNICEF) report about children situation in Malaysia, stated that in 2012 around 445,000 people with disabilities were registered voluntary in the social welfare Department or Jabatan Kebajikan Masyarakat (JKM) in Malay [2]. The number is bigger in other countries. For example, there are 11.9 million (19\% of population) disabled people in United Kingdom [3]. Studies indicate that globally, around $10 \%$ of the disabled people require a wheelchair [4]. Wheelchair is essential for people with mobility impairment to be able to move and get equal opportunities.

\section{WHEELCHAIR USERS IN MALAYSIA}

Malaysian legislation "Persons with Disabilities Act 2008" demonstrates an active role in the efforts of providing equal opportunity for the people with disabilities. However, a comprehensive investigation stated that many disabled people think that the "Persons with Disabilities Act 2008" is actually an administrative document, and disabled people are still discriminated against [1].

In fact, public facilities in Malaysia are not fully accessible by wheelchair users. For example, road designs are not supportive as walkable environments [5]. Consequently, it is not supportive for people of special needs. Therefore, the car is still the main way for transportation. Another example is the public parks. Basic physical needs for wheelchair users are provided in some parks, however not all the requirements are according to the standards [1]. Therefore, wheelchair users encounter different types of difficulties. Most of the time, they need assistance from others. Sometimes even with assistance, they still encounter difficulties and their safety is not guaranteed. There are a number of concerns related to providing proper accessibility for wheelchair users to public facilities. The concerns include budget, conflicts with infrastructure, natural factors, effectiveness of government documents, and lack of awareness. Malaysia is considered as a developing country. Therefore, the consideration of equal accessibility to outdoor environments in Malaysia is still lacking [1].

Apart from public facilities convenience accessibility, there are indications that only few number of people in need of wheelchairs have access to them. In addition, among those who can get a wheelchair, some of them are not able to get an appropriate one [4]. The lack of a proper wheelchair may lead to limitations in mobility and hence, the possibility of involvement in outdoor activities without help is decreased. Fig. 1 shows examples of cases where a suitable wheelchair is not available. The size of the wheelchair for the children in Fig. $1-\mathrm{a}$ is clearly not suitable. It is almost impossible for them to move without parent's help. The lady in Fig. 1-b has her four limbs lost. Unfortunately, she cannot get a proper Electric 
Powered Wheelchair (EPW) to move independently. Finally, Fig. 1-c shows all the wheelchairs available in one of the senior citizen nursing houses in Malaysia. We made a visit to this house in February 2017. The house was hosting 25 seniors during our visit time. 15 of them need a wheelchair. The functioning wheelchairs were 5 only. The lack of wheelchairs restricts the activities to be indoor only. There are hundreds of similar homes across Malaysia [6]. Most of the disabled and senior citizen homes are run by charities. Registered houses can get governmental support through JKM which covers food mainly.
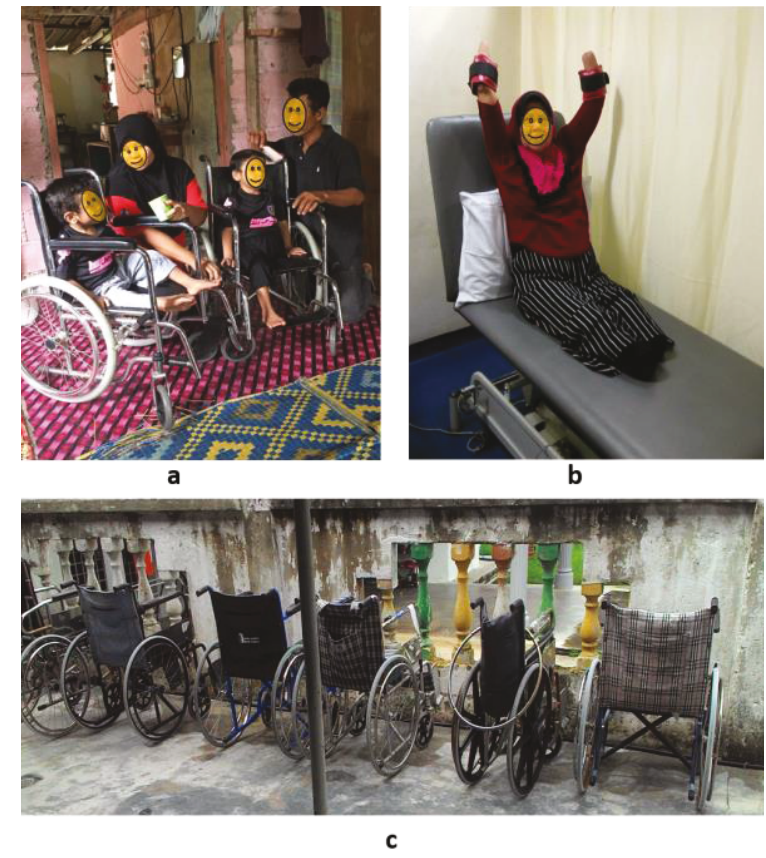

Fig. 1. Cases where a suitable wheelchair is not available.
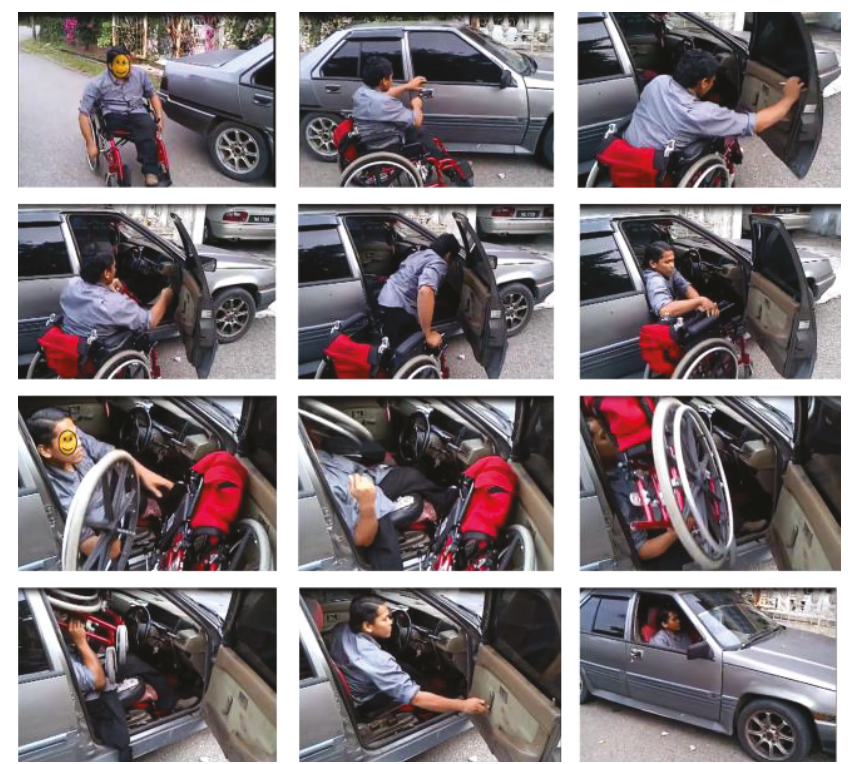

Fig. 2. The user prefers the manual wheelchair as he can independently fit it in the car.
Although having a good quality EPW seems to be a good solution for many cases, but in fact, people in Malaysia rarely use EPW. Most of the wheelchairs in Malaysia are imported. EPW market is not active and most of the imported wheelchairs are manual. A survey on wheelchairs in Malaysia was conducted by distributing a hardcopy questionnaire in several hospitals and by emailing the softcopy version of it. Moreover, we conducted interviews with the management of two disabled centers and one senior citizen center in three different cities in Malaysia. The results collected from the survey are to be published in a separate paper. However, according to our survey, wheelchair users tend to use wheelchairs independently. When asked about the preferable wheelchair type, if it is manual wheelchair or EPW, most of the participant said they prefer the EPW. Although EPWs are becoming more popular in many countries, they are very few in Malaysia. Moreover, the few EPW owners, still use the manual wheelchair besides to the EPW. A standard manual wheelchair weighs around $15-25 \mathrm{~kg}$ depending on the weight of the frame, components, and accessories. The lightest folding manual wheelchair is about $7-9 \mathrm{~kg}$. The problem is that cheap, typical models of EPW are bulky and weigh around 40 $\mathrm{kg}$ and above. The weight of a quality one is $20-30 \mathrm{~kg}$. Moreover, not all EPW models are user friendly. Most of them must disassembled part by part using tools like a screwdriver. On the other hand, newer models which are easier to handle and lighter are very expensive.

In fact, most of the users prefer to use the EPW but practically they cannot due to three main reasons:

1. The price of EPW is considered very expensive for many people in Malaysia, because the demand is law and units are imported by order.

2. Most of the Affordable EPWs are heavy and difficult to be folded and transported in car. Users need to use the car because roads designs in Malaysia are not supportive for people of special needs as mentioned earlier. Wheelchair users need to have a light foldable EPW. It might be surprising to know that some of the wheelchair users use a car independently but they do not use the EPW. Moreover, they prefer to use a manual wheelchair as shown in Fig. 2. Light EPWs which are designed to fit in the cars are expensive. It is more expensive than the user's car in Fig. 2.

3. People in Malaysia generally refrain from buying EPW due to the lack of maintenance and replacement parts specially the battery.

From the above discussion, we can conclude that innovative solutions are needed to improve the wheelchair users' accessibility to public facilities and to increase their ability to get involved in outdoor activities. Improving the facilities themselves to be suitable for wheelchair users may take long time and it is not easy due to the high budget needed. Many researchers have suggested advanced solutions which might be categorized under the title "smart wheelchair systems". Wheelchairs are considered smart systems according to: Human - machine interface, the navigation methods and 
other smart systems like safety driving and obstacle avoiding systems [7].

In this paper, a smart electric wheelchair based on add-on features is presented. The proposed system is an integration of different devices which can easily be installed to any manual wheelchair to convert it to a customized smart EPW.

\section{SYSTEM DESIGN}

To fulfill the requirements discussed in Section II, an innovative EPW design using add-on features is proposed. The general architecture of the smart EPW system is shown in Fig. 3. The system comprises two subsystems:

1. A conversion kit which comprises two electric wheel hub motors and a controlling system. The controlling system is designed to be flexible and to accept different types of controllers.

2. A health monitoring system which implements Internet of Things (IoT) features.

The kit design applies the plug-and-play concept. It takes in consideration decreasing the price, easiness of attaching and detaching, lightness and the transportability in any vehicle. The current prototype employs joystick and smartphone as controlling devices. It is extendable and can accept other addon devices to be developed in the future. Moreover, a special wheel holder is designed to guarantee the flexibility of attaching and detaching the controlling parts and the electric wheel motors. Further, it is compatible with different types of wheelchairs and different wheel sizes. The design is explained in details in the following sections.

\section{THE CONVERSION KIT}

Most of the available in market conversion kits comprise four main parts: The controlling interface which is usually a joystick, motor driver, two dc motors connected to two wheels and a battery. To make the design more compact, in the proposed system we use a brushless gearless hub motor with a built-in driver. The hub motor is shown in Fig. 4.

There are different designs for manual wheelchair with different wheel diameters. Similarly, different wheel hub motors diameters are available in the market. The big variety of the designs makes it difficult to design a universal conversion kit to be installed on any wheelchair. In case of size incompatibility, a modification on the original wheelchair chassis is needed. The proposed solution in this study is an adjustable wheel holder. The holder is designed to be compatible with the variety of sizes and types. The wheel hub motors used in our project are originally designed for an ebike. Therefore, the concept of a bicycle fork is utilized to design the proposed holder with some additional features.

The two wheel holders, must be able to withstand the weights of the wheelchair, the user, the battery and any expected add-on devices. The weight is estimated to be $120 \mathrm{~kg}$. Fig 5 shows a sketch of the wheel holder which comprises two parts: The wheel frame holder and an adjusting adapter. The wheel holder has multi holes to enable users to adjust the height according to the diameter of their original wheels.

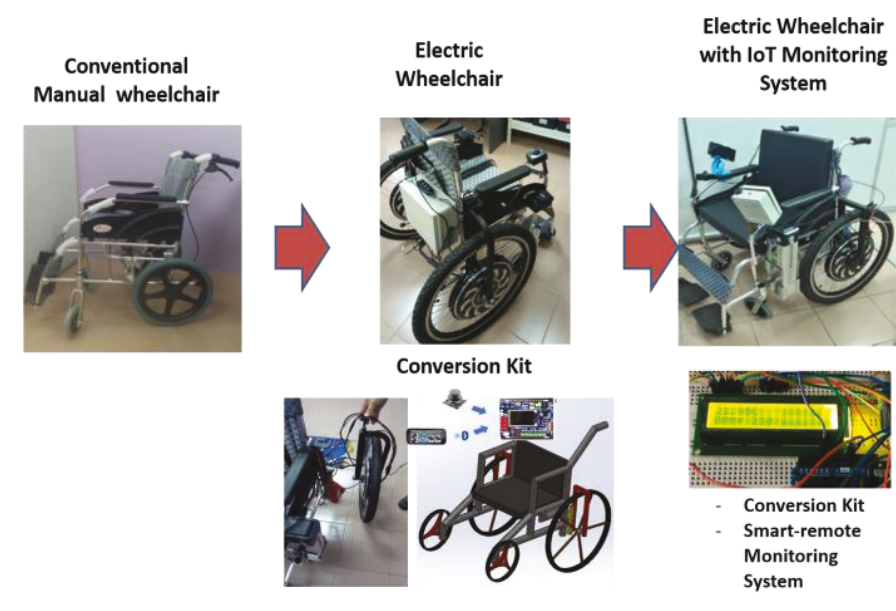

Fig. 3. The general smart EPW system.

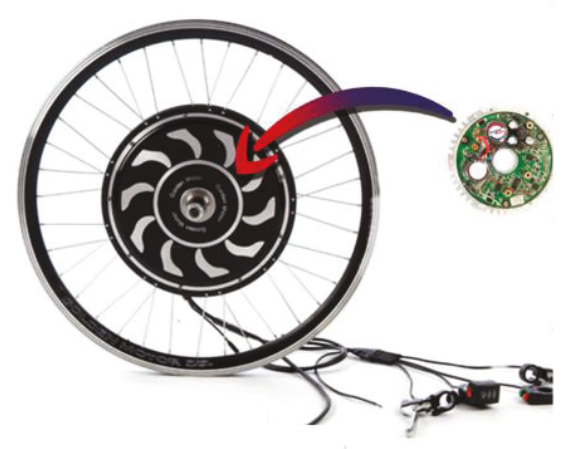

Fig. 4. The wheel hub motor which includes a built in driver [8].

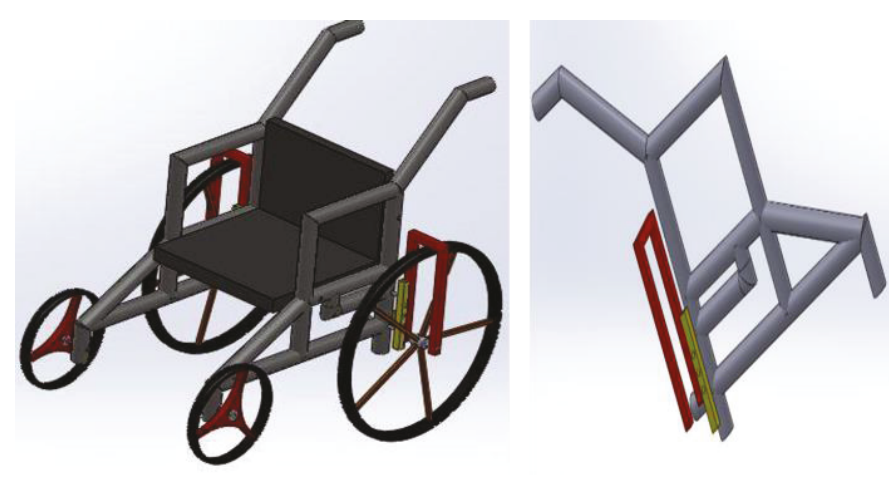

Fig. 5. The sketch of the wheel holder.

Hollow rectangle mild steel (1-inch $\times 1$-inch) was used to fabricate the holders. To ensure the wheel holder is stable and rigid enough to withstand the expected weight, a structural simulation on the design is carried out. The reported component stress and deformations are analyzed to validate the performance of the design as shown in Fig. 6. The simulation applies the usage of mild steel hollow rectangle as the material. From the analysis, it is found that the maximum displacement nodal magnitude is $0.271 \mathrm{~mm}$ located on the edge of the screw while the maximum stress is $87.17 \mathrm{~N} / \mathrm{mm}^{2}$ located at the 
welded parts between the screw and the wheel holder. The design is found to be safe as the stresses are within an acceptable range.

The controlling system is a main part in this design. Electric wheelchairs can be maneuvered by different types of controllers. The list includes joystick, head-arrays push-button controllers, Head motion, and eye tracking [7]. Joystick is the most popular device. However, some users cannot use the joystick properly due to many reasons such as the cases in Fig. 2. Android devices are very popular nowadays and can be used for many powerful controlling applications like robot operating, home appliances remote controlling, security, safety, and monitoring systems. In this project, the user can plug-andplay different types of controllers. The current prototype accepts joystick controller and a smartphone as an add-on option besides to the normal joystick. The smartphone can be used in two ways: Touch screen controller and gyroscope (tilt) controller. The joystick is connected directly to a Micro Controlling Unit (MCU). While HC-06 Bluetooth communication module is used to connect the smartphone with the MCU. The concept of add-on / plug-and-play is considered. Moreover, the system is extendable and can accept other controlling devices.

In the case of both controllers are connected simultaneously, the system considers the joystick as the default dominant controller. The system responds to the smartphone only in case the input from joystick is null.

Lithium Iron Phosphate (LiFePO4) 48V battery is used as a main power source for the hub motors. Moreover, LM2596HV circuit which is $3 \mathrm{~A}$ Step-down voltage regulator is used to supply $12 \mathrm{~V}$ power to the MCU.

\section{A. Controlling the wheelchair using the joystick}

According to the stick position of the joystick, two analogue signals are sent to the MCU. One signal is for horizontal axis and the other is for vertical axis. The MCU converts the position to speed and direction by sending a suitable signal to the hub motors through its built in driver.

The MCU used in this project is Atmega328P which is the MCU of the Arduino UNO platform. This MCU can only generate Pulse-Width Modulation (PWM) signals as analogue output. On the other hand, the hub motor, which is used in this project, has a built in driver which accepts DC analogue input. The original design is to use a throttle (potentiometer) to control the speed of an e-bike. Therefore, a PWM to DC converter circuit is used. First or second order RC filter can be used for this purpose. An experimental test was applied on first and second order RC filter to find the best values for linear output. An example of the output results is shown in Fig. 7. Based on the output results, the second order RC filter with two $1 \mathrm{~K} \Omega$ resistors and two $1 \mu \mathrm{F}$ capacitors is used. The PWM to analogue converter output is a dc signal. The output dc signal replaces the speed signal which is supposed to be produced by the throttle. The full controlling circuit is shown in Fig. 8 with the second order RC filter as part of it.
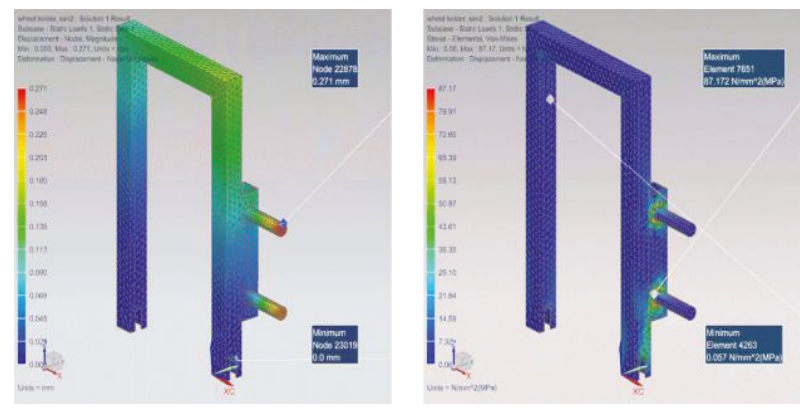

Fig. 6. Displacement and stress analysis results.

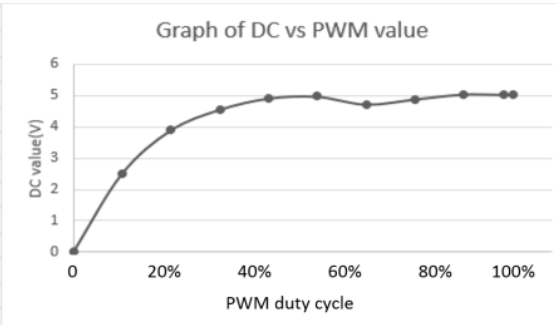

First order RC filter response

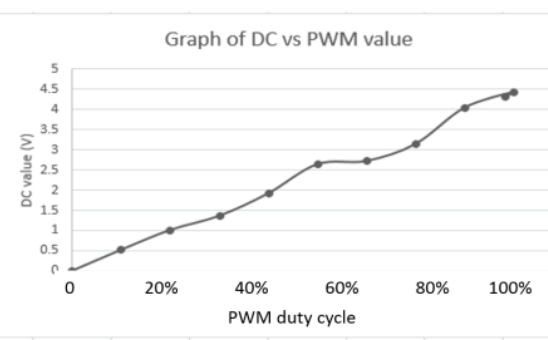

Second order RC filter response

Fig. 7. Comparison between first and second order RC filter for PWM to analogue converter.

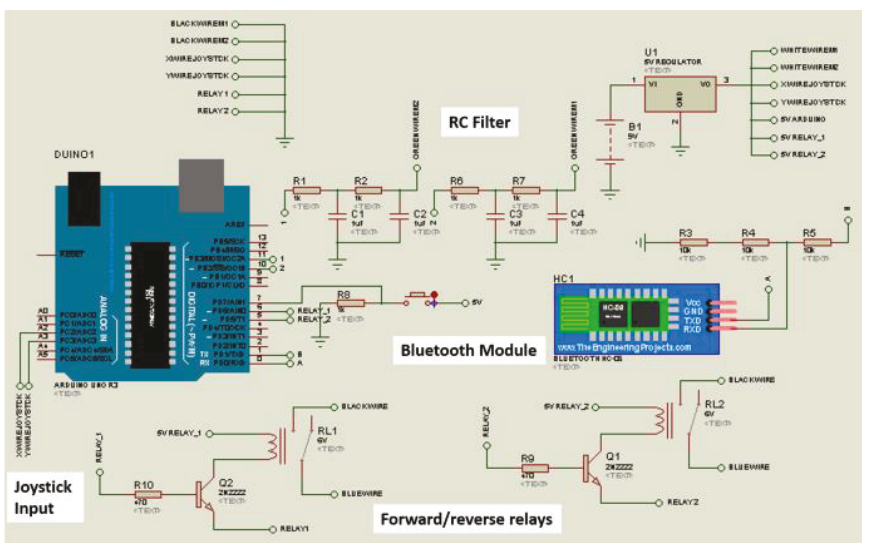

Fig. 8. Circuit diagram for the controlling part.

\section{B. Controlling the wheelchair using a smartphone}

A smartphone application (App) is used to send commands to the MCU through a Bluetooth channel. The application can be used in two ways: touch screen controller and gyroscope (tilt) controller. Fig. 9 shows one of the Android applications 
which can be used for the described functionality. In both cases commands are sent as texts which are received by the MCU using serial UART communication protocol. The MCU converts the commands to speed and direction by sending a suitable signal to the hub motors through its built in driver. The signal is sent through the same second order $\mathrm{RC}$ filter described in subsection (A). As mentioned earlier, the purpose of the filter is to convert the PWM signal to a dc signal which replaces the speed signal of the throttle. Bluetooth receiver is also shown as part of Fig. 8 .

The hub motor supports forward and reverse movement. Movement direction is controlled through the built-in driver. A relay is added to the circuit for each wheel to connect the wires which control the direction.

\section{Conversion kit weight aspects}

The weight of the proposed conversion kit is as following: $3.5 \mathrm{~kg}$ for battery $+2 \times 4.5 \mathrm{~kg}$ for the hub motors $+2 \times 1.5 \mathrm{~kg}$ for the adjustable wheel holder + less than $1 \mathrm{~kg}$ for the controlling and monitoring circuit elements. The total weight is $16.5 \mathrm{~kg}$ at maximum. Therefore, the total weight including the wheelchair is in the range of $23.5-41 \mathrm{~kg}$. However, even if a standard wheelchair is used, the conversion kit is significantly designed to allow users to disassemble the wheels and battery easily as shown in Fig. 3. Therefore, the user should have no problem in fitting it in a car independently.

\section{MONITORING SYSTEM}

Using a manual or EPW independently, rise up a problem which is the lack of patient monitoring. Wheelchair users may have health complaints and they are more subject to fatigue and weakness. Therefore, it is not safe to use a wheelchair independently. To solve the problem, a proposed add-on system aims to provide a remote monitoring for wheelchair users using the Internet of Things (IoT) concept.

Fig. 10 shows an overview diagram of the monitoring system. The proposed prototype comprises two sensors: a temperature and a heartbeat sensor. Fig. 11 shows the flowchart of the monitoring system. An MCU continuously reads the temperature and heart pulse rate of a user. Readings are shown on a Liquid Crystal Display (LCD). Cardiograph can be shown on a computer/laptop if it is connected to the system using simple USB cable. The system is designed to trigger an alarming sound if the condition of body temperature and/or heart pulses of a user are abnormal. At the same time, Short Message Service (SMS) and email are sent to people in charge of monitoring. The smartphone snaps a photo of the scene and attach it to the email. The email and the SMS are sent using Android application which communicate with the MCU of the monitoring system using Bluetooth. The application is also equipped with a database supporting utility which is used for logging data. The logged data are saved to the smartphone and simultaneously are sent to "dweet.io" cloud service. The data are provided for IoT "Freeboard" dashboards for online monitoring as shown in Fig. 12.

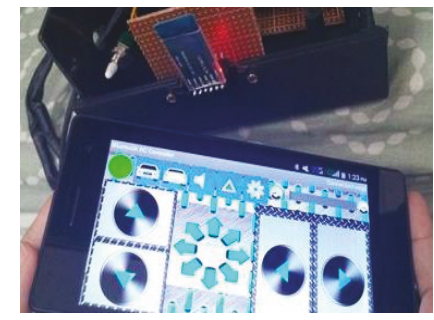

Fig. 9. The Android App which is used to control the wheelchair.

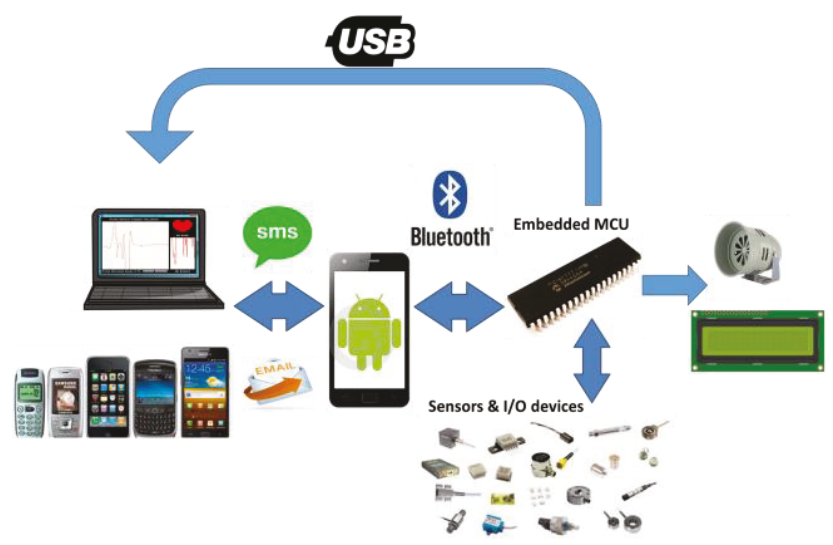

Fig. 10. The monitoring system overview diagram.

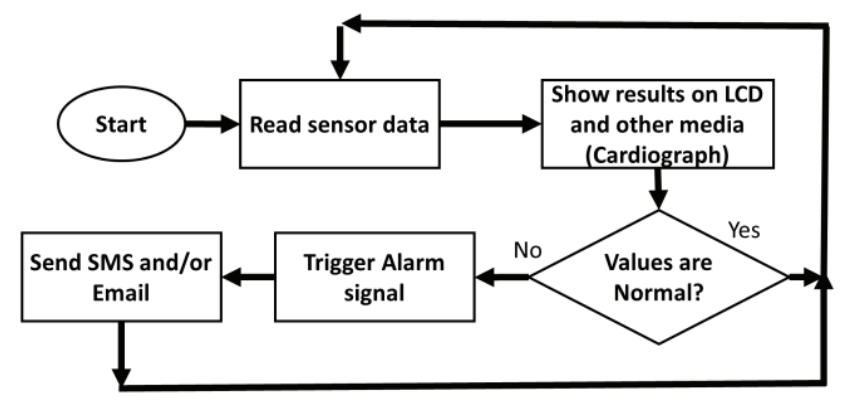

Fig. 11. Flowchart for the patient monitoring system.

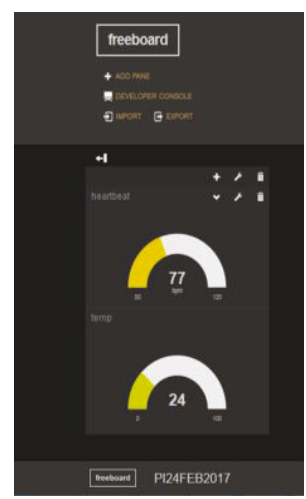

Fig. 12. "Freeboard" as an example of IoT dashboards.

Fig.13 shows the monitoring system circuit. The system can be installed to any wheelchair as an add-on device. Moreover, it can be powered from different batteries according to the main source battery of the hosting EPW. The flexibility is guaranteed through the $3 \mathrm{~A}$ step-down voltage regulator 
LM2596HV. In the proposed prototype, the monitoring system is powered directly from the $48 \mathrm{~V} \mathrm{LiFePO} 4$ battery.

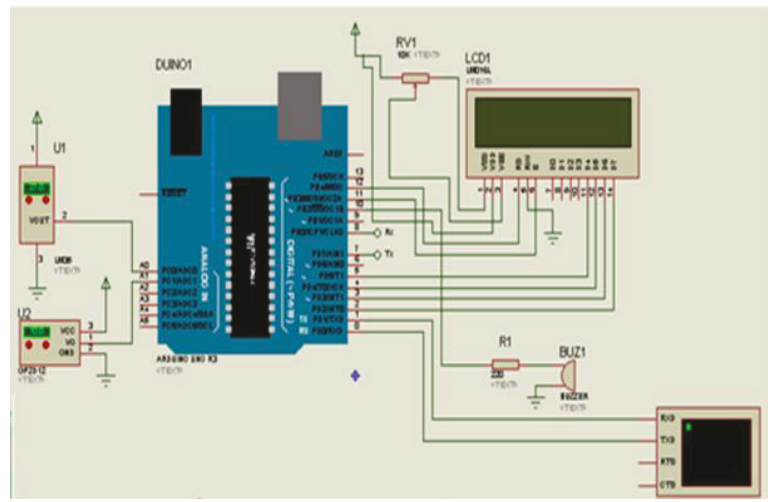

Fig. 13. Circuit diagram for the monitoring system.

\section{SYSTEMS TESTING RESULTS}

Fig. 14 shows the original wheelchair and the integrated EPW prototype including the monitoring system. The proposed system was implemented in real modes. The conversion kit is used to replace two 16 " wheels of the manual wheelchair in Fig. 14 by two 22" wheel hub motors. The system was tested and it moved smoothly using both the joystick and the smartphone. Both controlling methods of the smartphone i.e. the touch screen and the gyroscope (tilt) controller works perfectly. Response delay is less than 35 seconds.

To test the monitoring system, four participants helped in a testing experiment. The participants were asked to perform jogging for around 10 - 15 minutes. The heartbeat sensor and temperature sensors are used to measure their related vitalsigns before and after the jogging. The temperature $(\mathrm{T})$ is considered normal if $36^{\circ} \mathrm{C}<\mathrm{T} \leq 38^{\circ} \mathrm{C}$. Heartbeat rate $(\mathrm{Hb})$ is considered normal if $70 \mathrm{BPM}<\mathrm{Hb} \leq 90 \mathrm{BPM}$. After jogging the temperature and the heartbeat rate increases. Consequently, the alarm is triggered. Simultaneously, emails and SMSs are sent correctly and within one-minute after recognizing an abnormal situation. IoT monitoring dashboards are kept updated correctly with less than one-minute delay. These results validate the system as a real-time system.

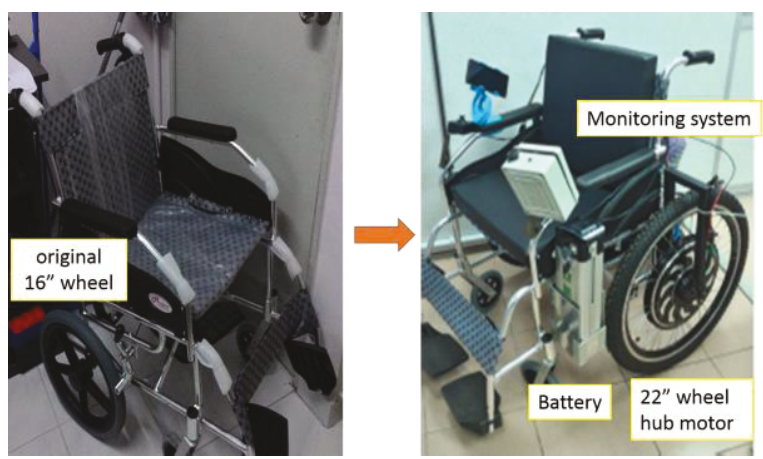

Fig. 14. The original wheelchair and the EPW prototype including the monitoring system.

\section{CONCLUSION}

This paper presents, an innovative design of an EPW conversion kit which meets the local requirements in Malaysia. The design is easy to use and improves the independent ability of using a wheelchair. A special design for an adjustable wheel holder was proposed. Two controlling devices are used, namely, a smartphone and a joystick. A health monitoring and alarming system which implements Internet of Things (IoT) features is installed as an add-on device. The EPW system is extendable and accepts other add-on devices. The system integrates Android smartphone applications, embedded MCUs and different sensors. A Bluetooth technology connection is used to exchange commands and data between the smartphone and MCU. All the devices can be powered from the EPW main power supply. The system was validated in real modes as a real-time system.

Future plans include adding more controlling device options, increasing the ability of the monitoring system to measure more vital-signs and improve its ability to analyze the data more efficiently. Moreover, more advanced add-on systems can be installed to the EPW like obstacle avoidance and rehabilitation devices.

\section{REFERENCES}

[1] L. Mohamed Yusof, "Accessibility for wheelchair users in Public Parks: A comparison between Adelaide and Kuantan," Master thesis, The University of Adelaide, 2010.

[2] UNICEF, "State of the world's children, situation in Malaysia, 2013: Children with disabilities," Available online: https://www.unicef.org/malaysia/sowc_sowc_13situation_in_malaysia.html (Last visit $\overline{8}, 201 \overline{7}$ )

[3] Papworth trust report, "Disability in the United Kingdom, facts and figures," 2016.

[4] World Health Organization (WHO), "Fact sheet on wheelchairs," $2010 . \quad$ Available online: http://apps.who.int/iris/handle/10665/205041 (Last visit 8, 2017)

[5] D. I. Azmi, H. A. Karim and M. Z. M. Amin, "Comparing the walking behaviour between urban and rural residents," Procedia - Social and Behavioral Sciences, Vol 68, pp. 406-416, 2012.

[6] Hati: Malaysia's largest open database of NGOs and social organizations. http://www.hati.my/ (Last visited: 8/2017).

[7] M. H. Alsibai and S. Abdul Manap, "A study on smart wheelchair systems," International Journal of Engineering Technology and Sciences (ijets) vol. 4 (1), pp. 25 - 35, 2015.

[8] GoldenMotor http://www.goldenmotor.com (Last visited: 8/2017) 